\title{
Frequency upconversion in $\mathrm{Er}^{3+}$-doped fluoroindate glasses pumped at $1.48 \mu \mathrm{m}$
}

\author{
G. S. Maciel and Cid B. de Araújo \\ Departamento de Física, Universidade Federal de Pernambuco, 50670-901 Recife, PE, Brazil \\ Y. Messaddeq \\ Departamento de Química, Universidade do Estado de Săo Paulo, 14800-900 Araraquara, SP, Brazil \\ M. A. Aegerter \\ Institut für Neue Materialien, Im Stadtwald, Gebaude 43, D66-123 Saarbrücken, Germany
}

(Received 16 July 1996; revised manuscript received 15 October 1996)

\begin{abstract}
We report on efficient frequency upconversion in $\mathrm{Er}^{3+}$-doped fluoroindate glass. The process is observed under $1.48 \mu \mathrm{m}$ laser diode excitation and results in fluorescence generation in the range from ultraviolet to

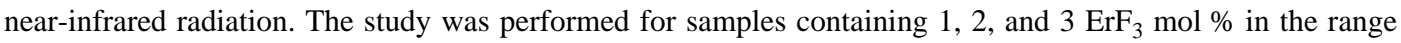
of temperatures from 24 to $448 \mathrm{~K}$. The upconverted signals were studied as a function of the laser intensity, and their dynamical behavior is described using a rate equation model which allows us to obtain the energy transfer rates between $\mathrm{Er}^{3+}$ ions in pairs and triads. [S0163-1829(97)07509-7]
\end{abstract}

\section{INTRODUCTION}

In the past years fluoride glasses doped with rare-earth (RE) ions have received great attention due to the possibilities of using these materials in numerous applications such as the operation of upconversion lasers, superfluorescent sources, and optical amplifiers, among others. ${ }^{1}$ Fluoride glasses are particularly attractive hosts because they can be fibered, maintaining a high intensity of pumping light over a long interaction length, and large RE concentrations can be incorporated easily into the host matrix. Furthermore, one of the advantages of fluoride hosts is the low energy of its more energetic phonons, which reduces the probability of multiphonon relaxation processes between the RE electronic levels.

Among the many fluoride compositions discovered, it was found recently that fluoroindate glasses may become one important material for photonics applications. The vitreous region in the system $\mathrm{InF}_{3}-\mathrm{ZnF}_{2}-\left(\mathrm{SrF}_{2}-\mathrm{BaF}_{2}\right)$ was established few years ago, ${ }^{2}$ and it was later observed that the glasses can be stabilized by addition of $\mathrm{GaF}_{3}, \mathrm{GdF}_{3}, \mathrm{CaF}_{2}$, and $\mathrm{NaF}^{3}{ }^{3}$ Previous works performed in this system include the study of their vibrational spectra and structure ${ }^{4}$ and the spectroscopy of samples doped with $\mathrm{Nd}^{3+}, \mathrm{Pr}^{3+}, \mathrm{Eu}^{3+}, 6$ and $\mathrm{Gd}^{3+} .6$ Our recent studies have shown that when doped with $\mathrm{Er}^{3+}, \mathrm{Nd}^{3+}$, and $\mathrm{Pr}^{3+}$, the fluoroindate glass presents large efficiency as upconverters from the infrared to visible ${ }^{7}$ and from orange to blue and violet. ${ }^{8,9}$

Erbium ions are appealing for spectroscopic investigation due to their energy level regular spacing which facilitates frequency upconversion via energy transfer or multistep pump absorption using a single excitation wavelength. Accordingly, studies of upconversion in $\mathrm{Er}^{3+}$-doped fluoride, ${ }^{10}$ borate,${ }^{11}$ tellurite and gallate, ${ }^{12}$ and fluorophosphate glasses ${ }^{13}$ have been reported. Presently, it is well known that the upconversion efficiency is larger for fluoride glasses because the multiphonon emission rates are much lower than the rates for the same levels of $\mathrm{Er}^{3+}$ in other glasses. ${ }^{1,14}$
In the present work we report results of our investigations on the upconversion properties of $\mathrm{Er}^{3+}$-doped fluoroindate glass using the infrared radiation from a cw diode laser as the excitation source. This work extends our previous roomtemperature studies ${ }^{7}$ for the whole range of temperatures from 24 to $448 \mathrm{~K}$.

\section{EXPERIMENTAL DETAILS}

The glasses studied have the following $\%$ molar composition: $\quad(39-x) \mathrm{InF}_{3}-20 \mathrm{ZnF}_{2}-16 \mathrm{BaF}_{2}-20 \mathrm{SrF}_{2}-2 \mathrm{GdF}_{3}-$ $2 \mathrm{NaF}-1 \mathrm{GaF}_{3}-x \mathrm{ErF}_{3} \quad(x=1,2,3)$. The samples preparation procedure is briefly described. $\mathrm{InF}_{3}$ was obtained by fluoration of $\operatorname{In}_{2} \mathrm{O}_{3}$ at $400{ }^{\circ} \mathrm{C}$ with $\mathrm{NH}_{4} \mathrm{~F}$ and $\mathrm{HF}$ in a platinum crucible. Then all fluoride components were mixed up and heated in a dry box under argon atmosphere at $700{ }^{\circ} \mathrm{C}$ for melting and $800{ }^{\circ} \mathrm{C}$ for finning. After this process the melt was poured and cooled into a preheated brass mold. The samples obtained have good optical quality, volumes of a few cubic centimeters, and they are nonhygroscopic.

Optical absorption spectra in the $200-800 \mathrm{~nm}$ range were obtained with a double-beam spectrophotometer, while the infrared spectra up to $1.8 \mu \mathrm{m}$ were measured with an optical spectrum analyzer.

Continuous-wave upconversion fluorescence measurements were performed using a diode laser emitting at 1.48 $\mu \mathrm{m}$ as the excitation source. The laser beam was chopped at $7 \mathrm{~Hz}$ and focused on the sample using a lens of $15 \mathrm{~cm}$ focal length. The sample fluorescence was collected perpendicularly to the direction of the incident beam and was dispersed by a $0.5-\mathrm{m}$ grating spectrometer. The signal was detected using either a GaAs or a $S 1$ photomultiplier, and it was sent to a lock-in amplifier or a digital oscilloscope connected to a personal computer for processing.

For the low-temperature measurements, the samples were mounted in a cold-finger Dewar with temperature measured by a thermocouple embedded in the mounting bracket. The temperature of the samples could be varied from 24 to $300 \mathrm{~K}$ 

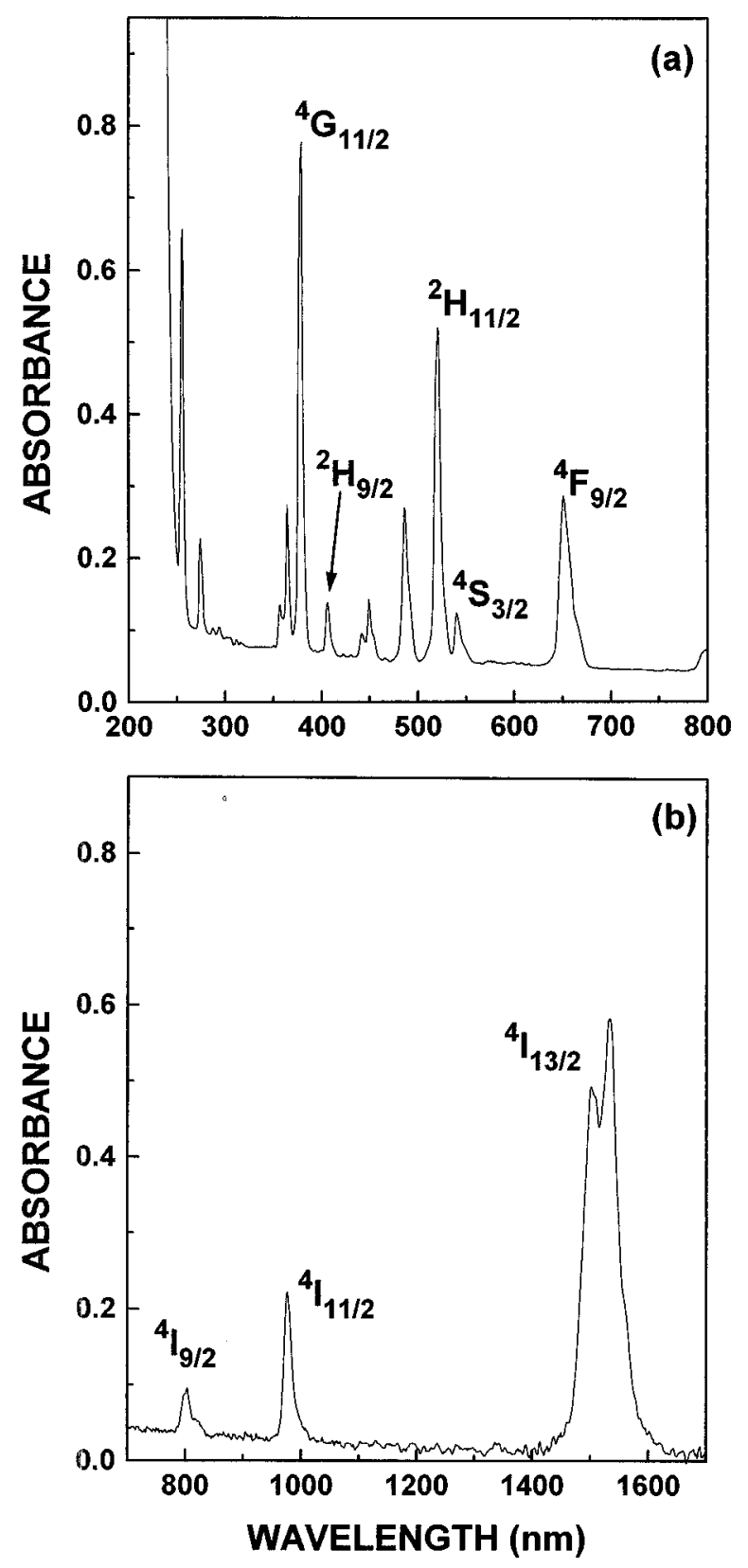

FIG. 1. Room-temperature absorbance spectra in the visible (a) and in the near-infrared (b) regions (sample with $x=3$, thickness 2.5 $\mathrm{mm})$.

with regulation of $0.1 \mathrm{~K}$. For the high-temperature experiments, the samples were warmed up to $448 \mathrm{~K}$ by a temperature-controlled thermal plate with regulation of $\pm 1 \mathrm{~K}$.

\section{RESULTS AND DISCUSSION}

Figure 1 shows the room-temperature absorption spectra in the visible range (a) and in the near infrared (b), obtained for one of the samples prepared. The broad features of several angstroms bandwidth can be identified with the transitions from the ground state $\left({ }^{4} I_{15 / 2}\right)$ to the excited states of the $\mathrm{Er}^{3+}$ ions. The bands observed at 274 and $256 \mathrm{~nm}$ are due to electronic transitions in the $\mathrm{Gd}^{3+}$ ions present in the glass matrix. No changes in the wavelengths of maxima were ob- served for the different concentrations used because the electronic transitions within the $4 f$ shell are not very sensitive to the crystalline field. The spectra obtained for the other samples are similar, except for the band intensities, which depend on the $\mathrm{Er}^{3+}$ concentration. The linewidths of all transitions are inhomogeneously broadened due to the site-to-site variation of the crystalline field strength.

Figure 2 shows the upconversion spectrum of the sample with $x=3$ under $5.6 \mathrm{~mW}$ excitation $\left(\sim 170 \mathrm{~W} / \mathrm{cm}^{2}\right)$ at room temperature. The diode laser excites resonantly the ${ }^{4} I_{15 / 2} \rightarrow{ }^{4} I_{13 / 2}$ transition and the observed emissions correspond to radiative transitions in the erbium ions from the excited states. The distinct emissions correspond to the transitions ${ }^{2} H_{9 / 2} \rightarrow{ }^{4} I_{15 / 2}(\sim 407 \mathrm{~nm}),{ }^{2} H_{11 / 2} \rightarrow{ }^{4} I_{15 / 2}(\sim 530 \mathrm{~nm})$, ${ }^{4} S_{3 / 2} \rightarrow{ }^{4} I_{15 / 2} \quad(\sim 550 \mathrm{~nm}), \quad{ }^{4} F_{9 / 2} \rightarrow{ }^{4} I_{15 / 2} \quad(\sim 670 \mathrm{~nm})$, ${ }^{4} I_{9 / 2} \rightarrow{ }^{4} I_{15 / 2}(\sim 808$ and $\sim 827 \mathrm{~nm}),{ }^{4} S_{3 / 2} \rightarrow{ }^{4} I_{13 / 2}(\sim 854 \mathrm{~nm})$, and ${ }^{4} I_{11 / 2} \rightarrow{ }^{4} I_{15 / 2}(\sim 980 \mathrm{~nm})$. The green and red transitions are readily visible by the naked eye. The spectra were analyzed with respect to their pump power dependence and temporal behavior. To analyze the results we first note that for unsaturated frequency upconversion the fluorescence signal $I_{S}$ will be proportional to some power $n$ of the excitation intensity such that $I_{S} \propto I^{n}$, where $n=2,3,4, \ldots$ is the number of infrared photons absorbed per upconverted photon emitted. The dependence of the fluorescence signal on the $1.48-\mu \mathrm{m}$ excitation intensity was such that $3.6<n<3.9$ for the emission at $407 \mathrm{~nm}, 2.4<n<2.7$ for the emission at 550 and $670 \mathrm{~nm}, 1.7<n<2.0$ for the emission at 808 and 827 $\mathrm{nm}, 2.4<n<3.3$ for the emission at $854 \mathrm{~nm}$, and $1.8<n<2.0$ for the emission at $980 \mathrm{~nm}$. The measurements were made for the three concentrations prepared, and the data for one of the samples are shown in Fig. 3. From the intensity dependence observed and the wavelength of the emitted radiations, we conclude that four laser photons are involved in the 1.48$\mu \mathrm{m}-$ to- $407-\mathrm{nm}$ conversion, three laser photons participate in the $1.48 \mu \mathrm{m}$-to-550-nm, $1.48-\mu \mathrm{m}$-to-670-nm, and 1.48 $\mu \mathrm{m}$-to-854-nm conversions, and two laser photons produce the $1.48-\mu \mathrm{m}$-to- $808-\mathrm{nm}, 1.48-\mu \mathrm{m}$-to-827-nm, and $1.48-\mu \mathrm{m}-$ to-980-nm frequency upconversions. The deviation from the exact $n$ values are due to strong absorption at $1.48 \mu \mathrm{m}$, the absorption of the upconverted fluorescence, and because the nonradiative decay from higher-lying states to fluorescent states may also contribute to the intensities of the observed spectral lines. The fluorescence line peaked at $980 \mathrm{~nm}$ was the most intense, being $\approx 50$ times more intense than the transition at $550 \mathrm{~nm}$. For an incident power of $5 \mathrm{~mW}$, about $1 \mu \mathrm{W}$ is converted into $980 \mathrm{~nm}$ emission. We also observed that for the sample with $x=3$ the signal at $980 \mathrm{~nm}$ was twice larger than for the sample with $x=2$ and 12 times larger than for the sample with $x=1$.

Different processes may lead to the population of highly excited $\mathrm{Er}^{3+}$ states after excitation in the near infrared $\left({ }^{4} I_{15 / 2} \rightarrow{ }^{4} I_{13 / 2}\right) .{ }^{13,15-19}$ These processes rely either on multistep excited state absorption (ESA) or energy transfer (ET) between $\mathrm{Er}^{3+}$ ion neighbors. The ET process, in which an excited ion nonradiatively transfers its energy to an already excited neighbor, is one of the most efficient mechanisms and has been observed in a large number of systems including fluoroindate glasses. ${ }^{8-14}$ This mechanism can arise from electric multipole or exchange interactions, and its rate occurrence depends on the RE concentration due to the ion-ion 

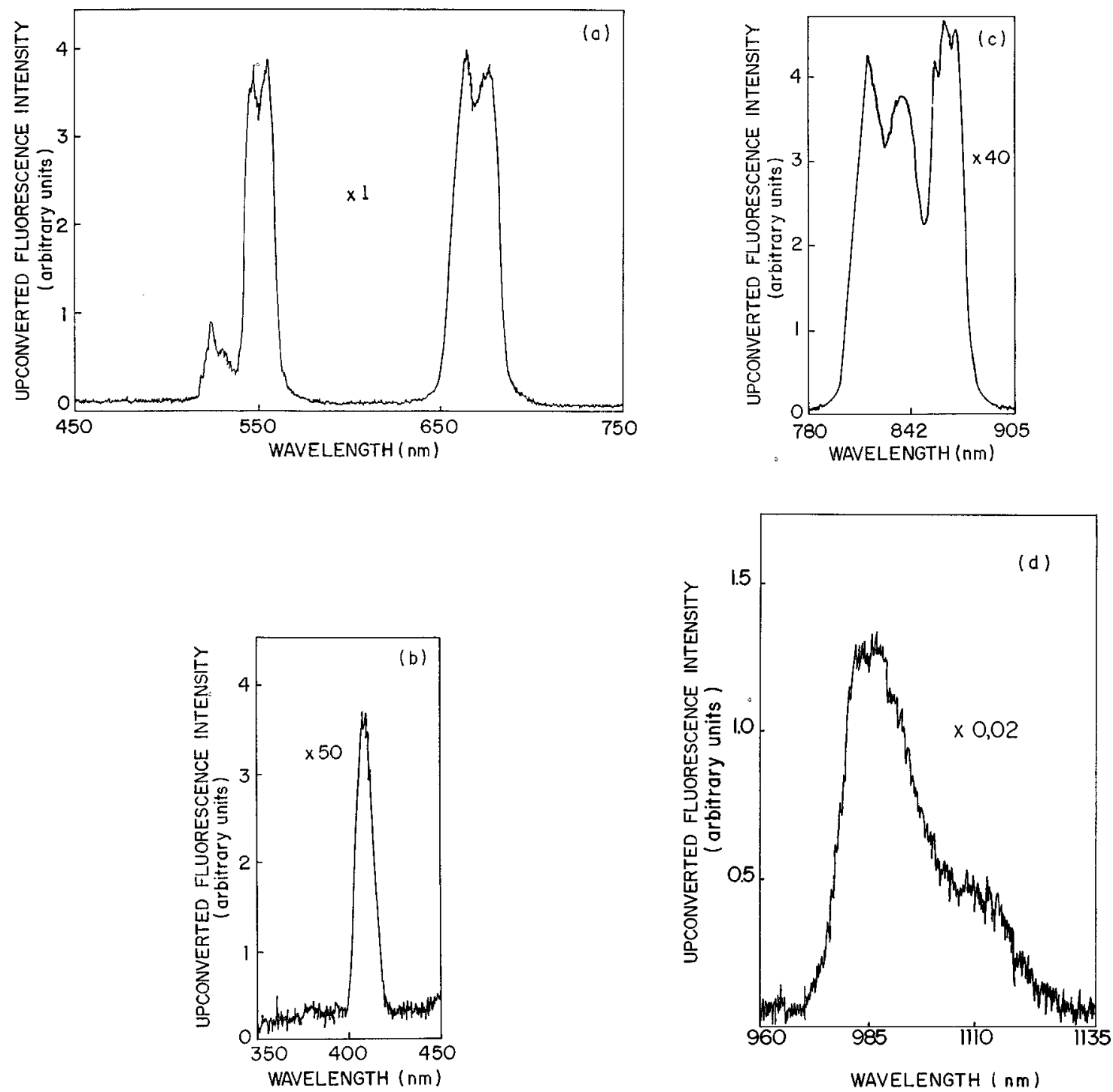

FIG. 2. Room-temperature upconversion fluorescence spectra (sample with $x=3$ ). The intensities of (b), (c), and (d) have been multiplied by 50,40 , and 0.02 , respectively.

separation. In the present case, we expect that ET is the dominant process because of the large $\mathrm{Er}^{3+}$ concentration in our samples and because the intermediate ESA step $\left({ }^{4} I_{13 / 2} \rightarrow{ }^{2} H_{11 / 2}\right)$ is a two-photon transition with small probability to occur due to the laser frequency detuning for intermediate states and the weak laser intensity used. Therefore, the most relevant pathway for upconversion initiates with the transition ${ }^{4} I_{15 / 2} \rightarrow{ }^{4} I_{13 / 2}$. Afterwards, ET between excited $\mathrm{Er}^{3+}$ ions at the ${ }^{4} I_{13 / 2}$ level will take one ion to the ${ }^{4} I_{9 / 2}$ level. This step is followed by other successive transfer processes from ions at the ${ }^{4} I_{13 / 2}$ state, which results in the excitation to higher levels. After nonradiative decay to lower states, radiative transitions to the ground state give rise to the observed upconverted fluorescence.

Figure 4 shows the relevant energy levels for the $4 f^{11}$ configuration of an $\mathrm{Er}^{3+}$ ion, together with two possible upconversion pathways and the observed fluorescence lines.

To characterize the temporal evolution of the upconverted signal, another series of experiments was performed. The laser beam was chopped at $7 \mathrm{~Hz}$, and the fluorescence was detected using a fast digital oscilloscope. The time resolution of the detection system was better than $1 \mathrm{~ms}$, and the signal corresponding to the various upconverted emissions grew to their maximum value in $\tau_{r}<15 \mathrm{~ms}$ and decay in $\tau_{d}<12 \mathrm{~ms}$. In general, the rise and decay times $\left(\tau_{r}\right.$ and $\left.\tau_{d}\right)$ decrease for increasing concentrations and for the range of $\mathrm{Er}^{3+}$ concentrations studied $\tau_{r}$ and $\tau_{d}$ change up to $50 \%$. The results obtained using a digital oscilloscope are indicated in Table I.

In order to understand the observed temporal behavior, we first recall that the upconversion efficiency depends on the probability of multistep excitation by ESA or by ET between adjacent excited ions, as well as the quantum efficiency of the emitting level. By either process, the dynamics of the upconversion signals depends on the lifetime of the intermediate excited states involved. For the samples used, the lifetime of the states ${ }^{2} H_{9 / 2},{ }^{4} S_{3 / 2},{ }^{4} F_{9 / 2}$, and ${ }^{4} I_{11 / 2}$ were reported in Ref. 18. The values obtained for the same range of $\mathrm{Er}^{3+}$ concentrations were $\left.\tau^{2}{ }^{2} H_{9 / 2}\right) \sim 15-20 \mu \mathrm{s}$, $\tau\left({ }^{4} S_{3 / 2}\right) \sim 84-573 \quad \mu \mathrm{s}, \quad \tau\left({ }^{4} F_{9 / 2}\right) \sim 302-645 \quad \mu \mathrm{s}, \quad \tau\left({ }^{4} I_{11 / 2}\right)$ $\sim 10.6-9.4 \mathrm{~ms}$, and $\tau\left({ }^{4} I_{13 / 2}\right) \sim 10.3 \mathrm{~ms}$. The lifetime of the 

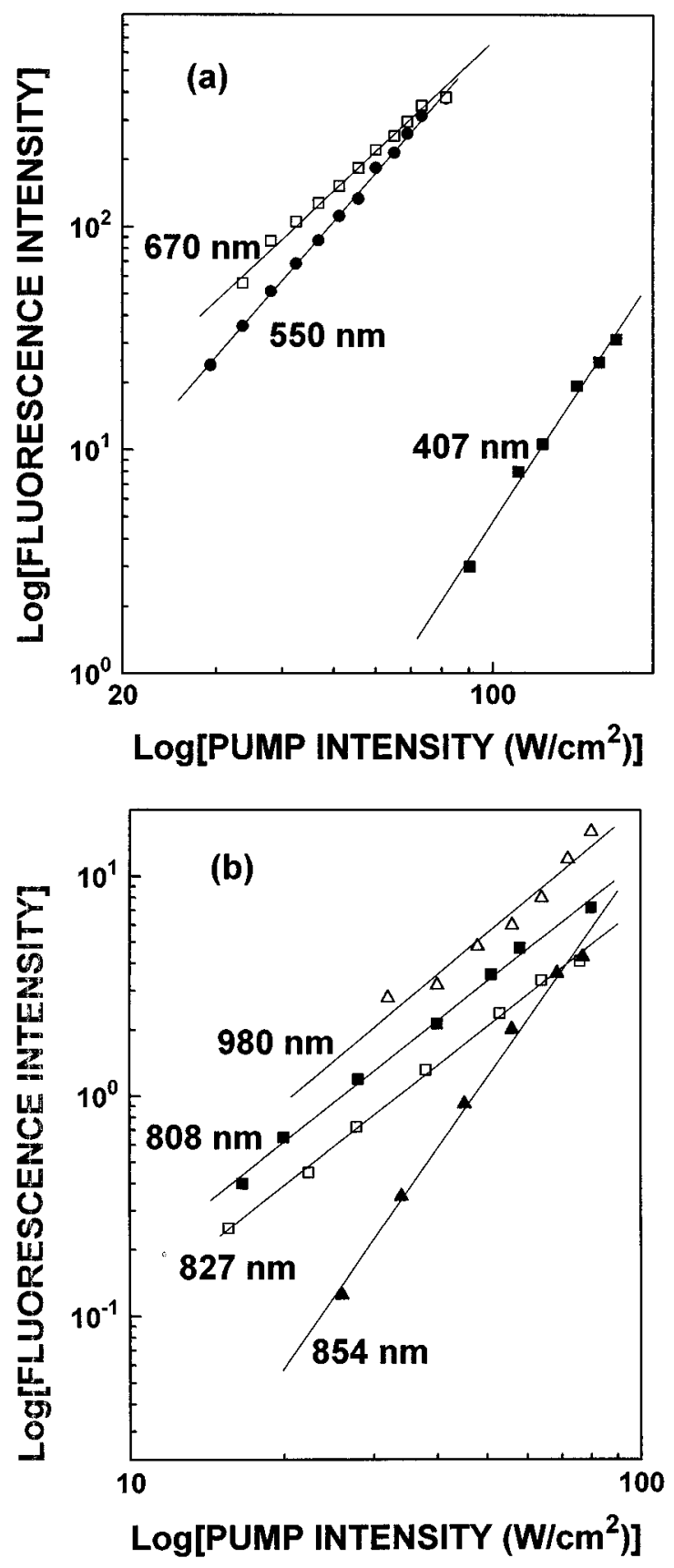

FIG. 3. Excitation intensity dependence of the upconversion fluorescence (sample with $x=2$ at room temperature). Straight lines with different slopes $n$ are obtained for each wavelength: (a) $n=3.6$ (407 nm), $n=2.7(550 \mathrm{~nm}), n=2.4(670 \mathrm{~nm})$; (b) $n=1.8(808 \mathrm{~nm})$, $n=1.8(827 \mathrm{~nm}), n=3.3(854 \mathrm{~nm}), n=1.9(980 \mathrm{~nm})$. The intensity corresponding to the $980-\mathrm{nm}$ line in (b) was scaled to be shown with the other infrared fluorescence lines observed.

state ${ }^{4} I_{9 / 2}$ was not measured, but on the basis of the known results for other host materials, we expect that its magnitude is $\approx 50 \mu \mathrm{s}$. The lifetimes are mainly determined by the multiphonon relaxation rates, which are small because of the small phonon energies associated with the fluoroindate matrix. ${ }^{4,10}$ Thus, considering that the states ${ }^{4} I_{9 / 2}$ and ${ }^{4} I_{11 / 2}$ are likely to participate in the upconversion processes, we conclude that the large values observed for $\tau_{r}$ and $\tau_{d}$ provide favorable evidence for the relevance of the ET mechanism.

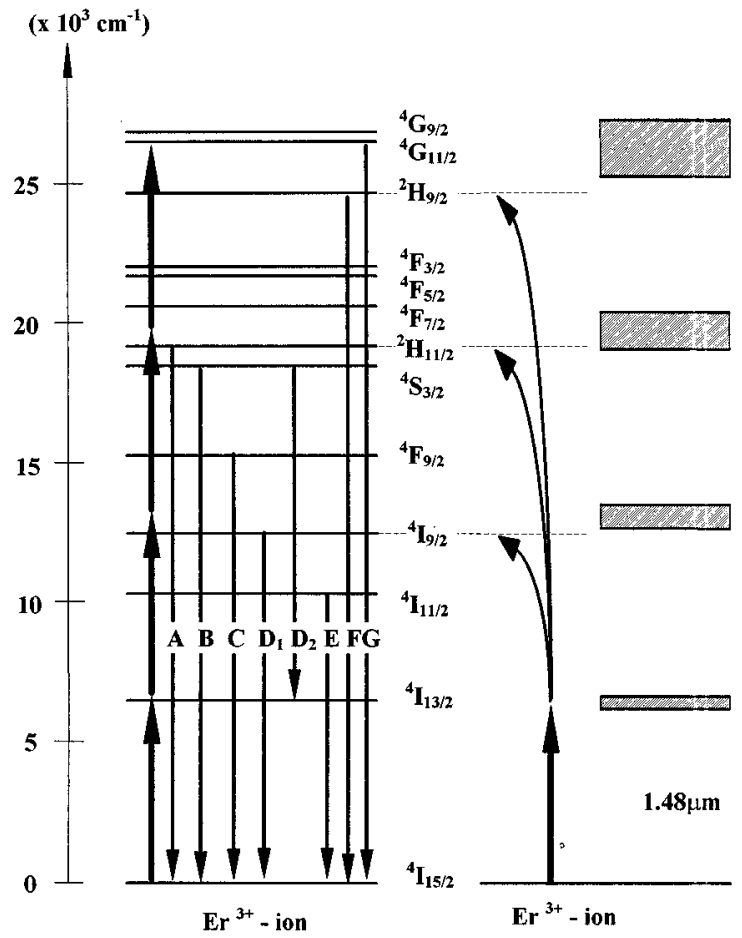

FIG. 4. Simplified energy scheme of $\mathrm{Er}^{3+}$ ion and excitation mechanisms. The curved arrows on the right stand for energy transfer. The letters beside the straight arrows correspond to the spectral lines $A(530 \mathrm{~nm}), B(550 \mathrm{~nm}), C(670 \mathrm{~nm}), D_{1}(808$ and $827 \mathrm{~nm})$, $D_{2}(854 \mathrm{~nm}), E(980 \mathrm{~nm}), F(407 \mathrm{~nm})$, and $G(377 \mathrm{~nm})$.

A simple description of the frequency upconversion dynamics can be obtained in terms of rate equations for the level populations. The equations can be written observing the following aspects: First, from the fact that the upconversion fluorescence intensity at 808,827 , and $980 \mathrm{~nm}$ increases quadratically with the laser intensity, we conclude that these emissions are due to the energy transfer process described by ${ }^{4} I_{13 / 2}+{ }^{4} I_{13 / 2} \rightarrow{ }^{4} I_{9 / 2}+{ }^{4} I_{15 / 2}+$ phonons, followed by a decay to level ${ }^{4} I_{11 / 2}$. The emissions observed are due to ${ }^{4} I_{9 / 2} \rightarrow{ }^{4} I_{15 / 2}(808$ and $827 \mathrm{~nm})$ and ${ }^{4} I_{11 / 2} \rightarrow{ }^{4} I_{15 / 2}(980 \mathrm{~nm})$. On the other hand, the cubic dependence of the fluorescence signals with laser intensity at 530, 550, 670, and $854 \mathrm{~nm}$ indicates that they are due to an energy transfer redistribution according to ${ }^{4} I_{13 / 2}+{ }^{4} I_{13 / 2}+{ }^{4} I_{13 / 2} \rightarrow{ }^{2} H_{11 / 2}+{ }^{4} I_{15 / 2}+{ }^{4} I_{15 / 2}$ +phonons, followed by nonradiative decay to levels ${ }^{4} S_{3 / 2}$ and ${ }^{4} F_{7 / 2}$. The lines at $407 \mathrm{~nm}\left({ }^{2} H_{9 / 2} \rightarrow{ }^{4} I_{15 / 2}\right)$ and $377 \mathrm{~nm}$ $\left({ }^{4} G_{9 / 2},{ }^{4} G_{11 / 2} \rightarrow{ }^{4} I_{15 / 2}\right)$ are due to energy transfer among four $\mathrm{Er}^{3+}$ ions in such a way that three ions deliver its energy to the fourth ion that is promoted to the states ${ }^{4} G_{9 / 2},{ }^{4} G_{11 / 2}$, and ${ }^{2} H_{9 / 2}$.

In order to estimate the energy transfer rates between $\mathrm{Er}^{3+}$ ions at room temperature, we used the following six-level rate equation system:

$$
\begin{aligned}
\frac{d n_{1}}{d t}= & -R_{12} n_{1}+W_{2} n_{2}^{2}+2 W_{3} n_{2}^{3}+\gamma_{21} n_{2} \\
& +\gamma_{31} n_{3}+\gamma_{41} n_{4}+\gamma_{51} n_{5}+\gamma_{61} n_{6},
\end{aligned}
$$


TABLE I. Rise and decay times measured for the frequency upconversion signals at room temperature.

\begin{tabular}{lcccccc}
\hline \hline & \multicolumn{2}{c}{$x=3$} & \multicolumn{2}{c}{$x=2$} & \multicolumn{2}{c}{$x=1$} \\
\cline { 2 - 7 }$\lambda(\mathrm{nm})$ & $\tau_{\text {rise }}(\mathrm{ms})$ & $\tau_{\text {decay }}(\mathrm{ms})$ & $\tau_{\text {rise }}(\mathrm{ms})$ & $\tau_{\text {decay }}(\mathrm{ms})$ & $\tau_{\text {rise }}(\mathrm{ms})$ & $\tau_{\text {decay }}(\mathrm{ms})$ \\
\hline 407 & 9.9 & 3.6 & 10.9 & 3.9 & 11.5 & 4.1 \\
550 & 8.1 & 4.5 & 9.9 & 4.9 & 13.0 & 5.6 \\
670 & 7.7 & 4.2 & 9.6 & 4.7 & 11.5 & 5.1 \\
808 & 5.2 & 4.3 & 6.8 & 4.9 & 7.7 & 5.4 \\
827 & 5.7 & 4.7 & 6.7 & 4.9 & 7.2 & 5.1 \\
854 & 7.8 & 5.6 & 10.6 & 5.8 & 15.0 & 6.0 \\
980 & 11.9 & 11.0 & 12.9 & 11.3 & 15.7 & 11.5 \\
\hline \hline
\end{tabular}

$$
\begin{aligned}
& \frac{d n_{2}}{d t}= R_{12} n_{1}-2 W_{2} n_{2}^{2}-3 W_{3} n_{2}^{3}-\gamma_{21} n_{2} \\
&+\gamma_{32} n_{3}+\gamma_{42} n_{4}+\gamma_{52} n_{5}+\gamma_{62} n_{6}, \\
& \frac{d n_{3}}{d t}=-\gamma_{3} n_{3}+\gamma_{43} n_{4}+\gamma_{53} n_{5}+\gamma_{63} n_{6}, \\
& \frac{d n_{4}}{d t}= W_{2} n_{2}^{2}-\gamma_{4} n_{4}+\gamma_{54} n_{5}+\gamma_{64} n_{6}, \\
& \frac{d n_{5}}{d t}=-\gamma_{5} n_{5}+\gamma_{65} n_{6} \\
& \frac{d n_{6}}{d t}=W_{3} n_{2}^{3}-\gamma_{6} n_{6}
\end{aligned}
$$

where 1 stands for level ${ }^{4} I_{15 / 2}, 2$ for ${ }^{4} I_{13 / 2}, 3$ for ${ }^{4} I_{11 / 2}, 4$ for ${ }^{4} I_{9 / 2}, 5$ for ${ }^{4} F_{9 / 2}$, and 6 for ${ }^{4} S_{3 / 2}$. Although energy transfer among triads at level ${ }^{4} I_{13 / 2}$, may promote one ion to the level ${ }^{4} H_{11 / 2}$, fast nonradiative decay (10 ns) populates the level ${ }^{4} S_{3 / 2}$. Thus we label in our model level 6 as the state ${ }^{4} S_{3 / 2}$. The contribution due to the population in level ${ }^{4} H_{9 / 2}$ was not considered because of the small intensity of the blue fluorescence which originates from that level. We also did not consider the nonresonant cross relaxation process $\left({ }^{4} I_{11 / 2}+{ }^{4} I_{13 / 2} \rightarrow{ }^{4} F_{9 / 2}+{ }^{4} I_{15 / 2}+\right.$ phonons $)$ because the contribution of this channel is assumed to be small in comparison with the resonant ET process between three ions. In the above rate equations, $R_{12}$ is the pump rate which is given by $\sigma I / h \nu$ where $\sigma=5.49 \times 10^{-25} \mathrm{~m}^{2}$ is the absorption cross section of the transition, $I=6.37 \times 10^{5} \mathrm{~W} / \mathrm{m}^{2}$ is the laser intensity, and $h \nu=1.34 \times 10^{-19} \mathrm{~J}$ is the photon energy. It is assumed that incident pump photons are resonant only with transitions from the ground state to the first excited state $\left({ }^{4} I_{13 / 2}\right)$. The quadratic terms $\left(W_{2} n_{2}^{2}\right.$ and $\left.-2 W_{2} n_{2}^{2}\right)$ and the cubic terms $\left(W_{3} n_{2}^{3}, 2 W_{3} n_{2}^{3}\right.$, and $\left.-3 W_{3} n_{2}^{3}\right)$ describe the energy transfer between two and three atoms, respectively. $W_{2}$ and $W_{3}$ are the pair and trio energy transfer rates between erbium ions, which are adjustable parameters in our model. The relaxation rate $\gamma_{i j}, i, j=1,2,3, \ldots$, is the radiative plus nonradiative decay rate from level $i$ to level $j$, and $\gamma_{i}$ is the total decay rate from level $i$. The radiative decays are obtained using Judd-Ofelt theory, ${ }^{20}$ and the nonradiative relaxation rates were obtained from fluorozirconate glass measurements. ${ }^{10}$ The actual values used are depicted in Table II. The software package MATHEMATICA was used to solve numerically the system of equations described above and to compare the results with the temporal behavior for $x=1$ and 3 . For a given value of $W_{2}$ and $W_{3}$, the theoretical dynamics of population was found in such a way that the fitting with the experimental data for every transition of our system could be obtained with the same set of parameters. The experimental rise times were very sensitive to the values of $W_{2}$ and $W_{3}$, while the decay times were dependent almost exclusively on the level lifetimes. The results obtained for $W_{2}$ and $W_{3}$ are depicted in Table II. The values of $\mathcal{W}_{2}\left(\mathcal{W}_{3}\right)$ in Table III were obtained from $W_{2}\left(W_{3}\right)$ divided by the concentration (squared concentration) of erbium ions, in order to compare with values reported in the literature. Note that $W_{2}$ and $W_{3}$ have the same order of magnitude than most $\gamma_{i j}$. Figure 5 shows the experimental results together with the theoretical curves for samples with $x=1$ and 3 .

The temperature variation of the upconversion fluorescence was studied from 24 to $448 \mathrm{~K}$ for one of the samples $(x=3)$, and the results are presented below. In Fig. 6(a) it is shown that the integrated fluorescence intensity at 407, 550,

TABLE II. Values of decay rates used and fitting parameters $\left(W_{2}\right.$ and $\left.W_{3}\right)$.

\begin{tabular}{lcc}
\hline \hline & $x=1$ & $x=3$ \\
\hline$\gamma_{21}\left(\mathrm{~s}^{-1}\right)$ & 100 & 115.3 \\
$\gamma_{31}\left(\mathrm{~s}^{-1}\right)$ & 79 & 95.76 \\
$\gamma_{32}\left(\mathrm{~s}^{-1}\right)$ & 13 & 13.62 \\
$\gamma_{41}\left(\mathrm{~s}^{-1}\right)$ & 159.3 & 116.72 \\
$\gamma_{42}\left(\mathrm{~s}^{-1}\right)$ & 29.3 & 35.70 \\
$\gamma_{43}\left(\mathrm{~s}^{-1}\right)$ & $3 \times 10^{5}$ & $3 \times 10^{5}$ \\
$\gamma_{51}\left(\mathrm{~s}^{-1}\right)$ & 1307.2 & 1115 \\
$\gamma_{52}\left(\mathrm{~s}^{-1}\right)$ & 243 & 206.41 \\
$\gamma_{53}\left(\mathrm{~s}^{-1}\right)$ & 36.9 & 43.60 \\
$\gamma_{54}\left(\mathrm{~s}^{-1}\right)$ & $3 \times 10^{3}$ & $3 \times 10^{3}$ \\
$\gamma_{61}\left(\mathrm{~s}^{-1}\right)$ & 674.1 & 836.62 \\
$\gamma_{62}\left(\mathrm{~s}^{-1}\right)$ & 180.1 & 223.1 \\
$\gamma_{63}\left(\mathrm{~s}^{-1}\right)$ & 59.8 & 74.14 \\
$\gamma_{64}\left(\mathrm{~s}^{-1}\right)$ & 22.1 & 28.01 \\
$\gamma_{65}\left(\mathrm{~s}^{-1}\right)$ & 400 & 400 \\
$R_{12}\left(\mathrm{~s}^{-1}\right)$ & 2.6 & 2.6 \\
$W_{2}\left(\mathrm{~s}^{-1}\right)$ & 800 & 1000 \\
$W_{3}\left(\mathrm{~s}^{-1}\right)$ & 300 & 500 \\
\hline \hline
\end{tabular}


TABLE III. Energy-transfer-rate values for $\mathrm{Er}^{3+}$ in different hosts.

\begin{tabular}{lcccc}
\hline \hline $\mathcal{W}_{2}\left(\mathrm{~cm}^{3} / \mathrm{s}\right)$ & $\mathcal{W}_{3}\left(\mathrm{~cm}^{6} / \mathrm{s}\right)$ & Host matrix & Concentration & Reference \\
\hline $1.5 \times 10^{-17}$ & & YAG & $16.6 \%$ & 21 \\
$1 \times 10^{-16}$ & silica fiber & $600 \mathrm{ppm}$ & 22 \\
$2 \times 10^{-17}$ & YAlO & $10 \%$ & 23 \\
$3.51-1.47 \times 10^{-18}$ & $5.77-1.08 \times 10^{-39}$ & fluoroindate glass & $1 \%-3 \%$ & Present work \\
\hline \hline
\end{tabular}

and $670 \mathrm{~nm}$ decreases with increasing temperature in the range from room temperature to $433 \mathrm{~K}$. We note that in this temperature interval the line at $670 \mathrm{~nm}$ shows a slower decrease of population with increasing temperature when com-
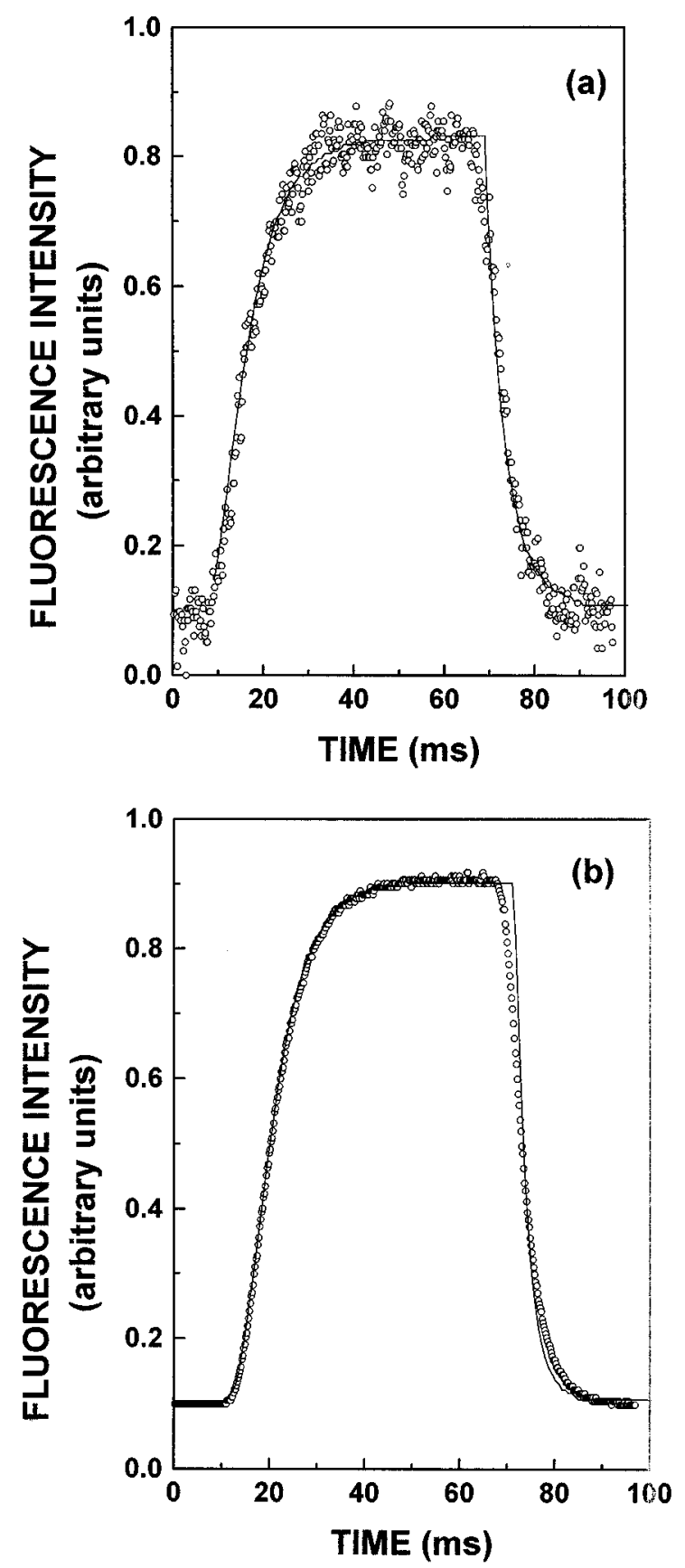

FIG. 5. Temporal behavior of the upconversion signal (a) emission at $808 \mathrm{~nm}(x=1)$ and (b) emission at $550 \mathrm{~nm}(x=3)$. pared with the lines at 550 and $407 \mathrm{~nm}$. When the temperature of the sample is lowered to below $300 \mathrm{~K}$, the upconverted fluorescence intensity increases by one order of magnitude, and another emission peaked at $377 \mathrm{~nm}$, corresponding to ${ }^{4} G_{11 / 2} \rightarrow{ }^{4} I_{15 / 2}$ transition becomes detectable, but its intensity is $\approx 10^{4}$ times weaker than the $550 \mathrm{~nm}$ emission, at $24 \mathrm{~K}$. The relative intensities of the emission lines are changed with respect to room temperature. For example, the green line emitted from state ${ }^{4} S_{3 / 2}(\sim 550 \mathrm{~nm})$ becomes so strong that the windows of the Dewar are illuminated when the room lights are off. On the other hand, the transition ${ }^{2} H_{11 / 2} \rightarrow{ }^{4} I_{15 / 2}$ at $\sim 530 \mathrm{~nm}$ was not observed for temperatures below $160 \mathrm{~K}$. The results obtained are summarized in Fig. 6(b), which shows the temperature dependence of the frequency upconversion signals at $\sim 377, \sim 407, \sim 550$, and $\sim 670 \mathrm{~nm}$. For all lines the ratio between the intensity for a given temperature with respect to the intensity at $295 \mathrm{~K}$ decreased when the temperature is increased above $50 \mathrm{~K}$. However, below $50 \mathrm{~K}$ an increase of the signal intensity for all the lines in the range $24-50 \mathrm{~K}$ is observed. This behavior at low temperature indicates that population thermalization does not occur below $50 \mathrm{~K}$. We also observe that for temperatures above $50 \mathrm{~K}$ the population temperature behavior is similar to the one described in Fig. 6(a). In Fig. 6(c) we show the ratio between the integrated intensities of light emitted at 670 and $550 \mathrm{~nm}$. It can be observed that above $230 \mathrm{~K}$ the red emission becomes stronger because of the significative multiphonon relaxation from the ${ }^{4} S_{3 / 2}$ state to the state ${ }^{4} F_{9 / 2}$. Another interesting result is that the relative intensity between the two lines at $\sim 530$ and $\sim 550 \mathrm{~nm}$ changes with temperature, which reveals the efficient thermal coupling between the excited states ${ }^{2} H_{11 / 2}$ and ${ }^{4} S_{3 / 2}$. Because of the small energy gap between those levels $\left(\sim 750 \mathrm{~cm}^{-1}\right)$, the state ${ }^{2} H_{11 / 2}$ may be populated from ${ }^{4} S_{3 / 2}$ by thermal excitation and a quasithermal equilibrium occurs between the two levels. This temperature behavior is consistent with previous reports for fluoride glasses, ${ }^{10}$ but in the present case the frequency upconversion process is so efficient that the fluoroindate glass could be used to build a sensitive temperature sensor. $^{15}$

\section{CONCLUSION}

We have observed efficient frequency upconversion from infrared to visible and to ultraviolet in $\mathrm{Er}^{3+}$-doped fluoroindate glass. The analysis of the emitted radiation indicates that the dominant mechanism involved in the upconversion process is the energy transfer among excited erbium ions. The large energy transfer rates observed are probably due to dipole-dipole interactions, but in order to extend our knowledge regarding the nature of the interactions among $\mathrm{Er}^{3+}$ 

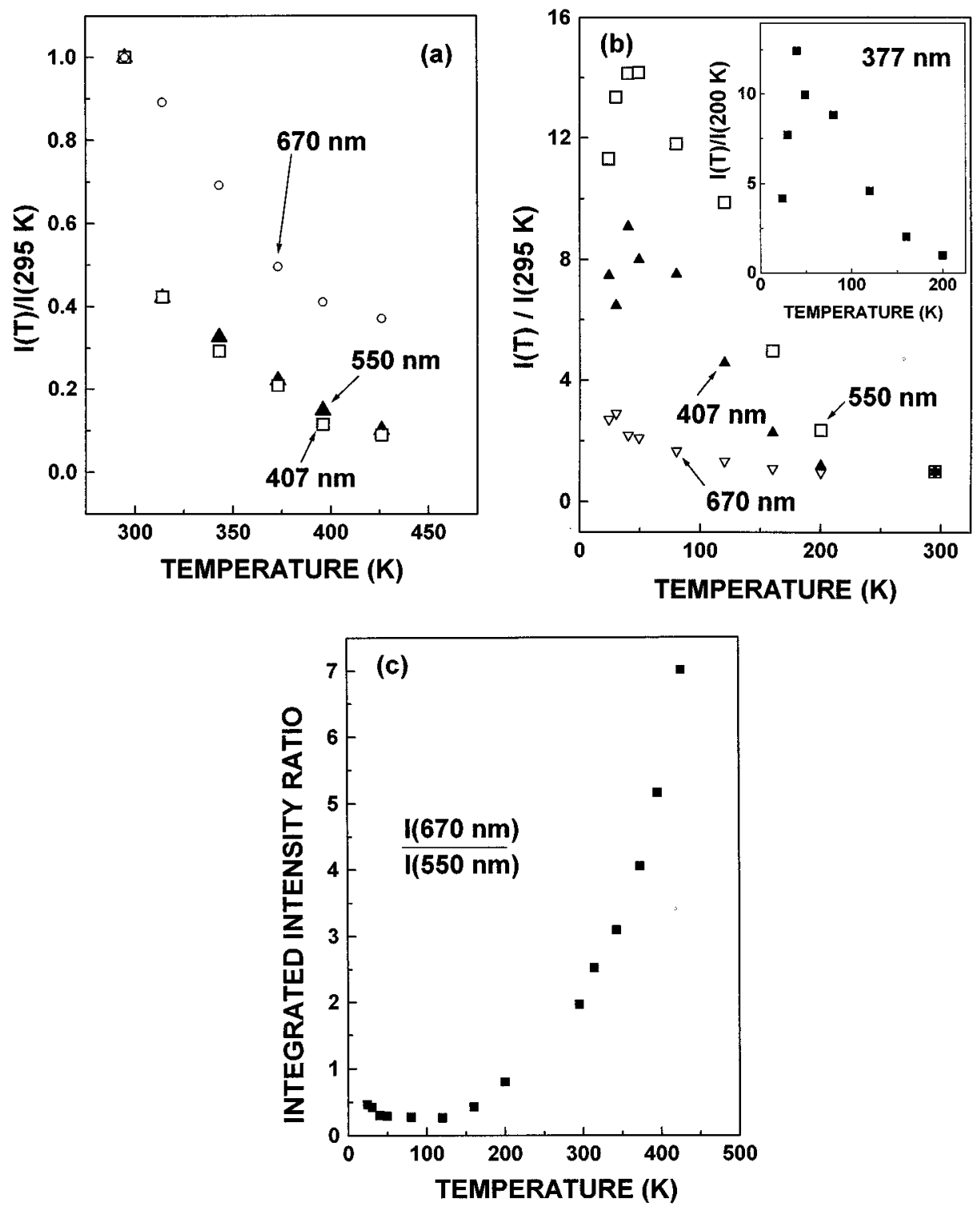

FIG. 6. Temperature dependence of the frequency upconversion integrated intensity at 407, 550, and $670 \mathrm{~nm}$ (a) for temperatures above $298 \mathrm{~K}$ and (b) for temperatures up to $298 \mathrm{~K}$. The inset shows the behavior of the anti-Stokes signal at $377 \mathrm{~nm}$. (c) Dependence of the frequency upconversion integrated intensity ratio between the lines at 550 and $670 \mathrm{~nm}$ as a function of temperature (sample with $x=3$ ).

ions in this material, further investigation will be undertaken in the future.

\section{ACKNOWLEDGMENTS}

This work was supported by the Brazilian Agencies Conselho Nacional de Desenvolvimento Científico e Tecno- lógico (CNPq), Financiadora Nacional de Estudos e Projetos (FINEP), Fundação Coordenação de Aperfeiçoamento de Pessoal de Ensino Superior (CAPES), and Fundação de Amparo à Ciência e Tecnologia (FACEPE). We thank also B. J. P. da Silva for polishing the samples and TELEBRAS for the loan of the diode laser used.
${ }^{1}$ See, for example, Rare-Earth Doped Fiber Lasers and Amplifiers, edited by M. J. Digonnet (Dekker, New York, 1993), and references therein.

${ }^{2}$ Y. Messaddeq and M. Poulain, Mater. Sci. Forum 67-68, 161 (1989).

${ }^{3}$ Y. Messaddeq, A. Delben, M. A. Aegerter, A. Soufiane, and M. Poulain, J. Non-Cryst. Solids 161, 210 (1993); J. Mater. Res. 8, 885 (1993).
${ }^{4}$ R. M. Almeida, J. C. Pereira, Y. Messaddeq, and M. A. Aegerter, J. Non-Cryst. Solids 161, 105 (1993).

${ }^{5}$ C. X. Cardoso, Y. Messaddeq, L. A. Nunes, and M. A. Aegerter, J. Non-Cryst. Solids 161, 277 (1993).

${ }^{6}$ S. J. L. Ribeiro, R. E. O. Diniz, Y. Messaddeq, L. A. O. Nunes, and M. A. Aegerter, Chem. Phys. Lett. 220, 214 (1994).

${ }^{7}$ Cid B. de Araújo, L. de S. Menezes, G. S. Maciel, L. H. Acioli, A. S. L. Gomes, Y. Messaddeq, A. Florez, and M. A. Aegerter, 
Appl. Phys. Lett. 68, 602 (1996).

${ }^{8}$ L. E. E. de Araújo, A. S. L. Gomes, Cid B. de Araújo, Y. Messaddeq, A. Florez, and M. A. Aegerter, Phys. Rev. B 50, 16219 (1994).

${ }^{9}$ L. de S. Menezes, Cid B. de Araújo, Y. Messaddeq, and M. A. Aegerter, J. Non-Cryst. Solids (to be published).

${ }^{10}$ D. C. Yeh, W. A. Sibley, M. Suscavage, and M. G. Drexhage, J. Appl. Phys. 62, 266 (1987); M. D. Shinn, W. A. Sibley, M. G. Drexhage, and R. N. Brown, Phys. Rev. B 27, 6635 (1983); K. Hirao, S. Todoroki, and N. Soga, J. Non-Cryst. Solids 43, 40 (1992).

${ }^{11}$ S. Tanabe, T. Ohyagi, N. Soga, and T. Hamada, Phys. Rev. B 46, 3305 (1992).

${ }^{12}$ S. Tanabe, K. Hirao, and N. Soga, J. Non-Cryst. Solids 122, 79 (1990)

${ }^{13}$ S. Tanabe, S. Yoshii, K. Hirao, and N. Soga, Phys. Rev. B 45, 4620 (1992).

${ }^{14}$ X. Zou and T. Izumitani, J. Non-Cryst. Solids 162, 68 (1993).

${ }^{15}$ G. S. Maciel, L. de S. Menezes, A. S. L. Gomes, Cid B. de
Araújo, Y. Messaddeq, A. Florez, and M. A. Aegerter, IEEE Photonics Technol. Lett. 7, 1474 (1995).

${ }^{16}$ B. R. Reddy and P. Venkateswarlu, Appl. Phys. Lett. 64, 1327 (1994).

${ }^{17}$ M. P. Hehlen, G. Frei, and F. U. Güdel, Phys. Rev. B 50, 16264 (1994).

${ }^{18}$ R. Reiche, L. A. O. Nunes, C. C. Carvalho, Y. Messaddeq, and M. A. Aegerter, Solid State Commun. 85, 773 (1993); T. Catunda, L. A. O. Nunes, A. Florez, Y. Messaddeq, and M. A. Aegerter, Phys. Rev. B 53, 6065 (1996).

${ }^{19}$ P. Xie and S. C. Rand, Appl. Phys. Lett. 63, 3125 (1993).

${ }^{20}$ B. R. Judd, Phys. Rev. 127, 750 (1962); G. S. Ofelt, J. Chem. Phys. 37, 511 (1962).

${ }^{21}$ W. Q. Shi, M. Bass, and M. Birnbaum, J. Opt. Soc. Am. B 7, 1456 (1990).

${ }^{22}$ P. Blixt, J. Niisson, T. Carlnas, and J. B. Jaskorzynska, IEEE Photonics Technol. Lett. 3, 996 (1991).

${ }^{23}$ D. J. Simkin, J. A. Koningstein, P. Myslinski, S. A. Boothroyd, and J. Chrostowski, J. Appl. Phys. 73, 8046 (1993). 